\title{
Analyzing Direct Non-local Dependencies in Attribute Grammars
}

\author{
John Tang Boyland \\ Computer Science Department, Carnegie Mellon University \\ 5000 Forbes Avenue; Pittsburgh, PA 15213-3891 \\ john. boylandeacm.org
}

\begin{abstract}
Describing the static semantics of programming languages with attribute grammars is eased when the formalism allows direct dependencies to be induced between rules for nodes arbitrarily far away in the tree. Such direct non-local dependencies cannot be analyzed using classical methods, which enable efficient evaluation.

This paper presents a new technique for analyzing such dependencies. Attribute grammars are extended to permit references to objects with fields to be passed through the attribute system. Fields may be read and written through these references. The extension has a declarative semantics in the spirit of classical attribute grammars.

This paper describes a technique for rendering the direct non-local dependencies in classical terms, after which well-known attribute grammar scheduling algorithms may be used. The generated classical dependencies use control attributes, attributes used only for scheduling and which involve no run-time computation. Thus the resulting implementations can be efficient: reading a field can be implemented as a simple indirect load; writing a field as an indirect store.
\end{abstract}

\section{Introduction}

Attribute grammars [13] were developed to specify the semantics of programming languages as an alternative to writing a compiler in an imperative language. An imperative program is not an ideal specification, because it expresses how a computation is to proceed, rather than what the desired result is to be. A more declarative approach is to specify what values need to be computed. Such a specification can be executed after first (automatically) determining an evaluation order that respects the dependencies in the specification. An advantage of declarative specifications is that they can be incrementalized by simply re-evaluating changed values. Incrementalizing an imperative program is much more difficult; maintaining a copy of every version of the state is impractical. Attribute grammars take the declarative approach; an attribute grammar specifies a set of attribute equations for each production in a context-free grammar. In the definition of classical attribute grammars, attribute values are specified as pure functions of attribute values of neighboring nodes. A variety of implementation techniques have been developed [1] and several usable attribute grammar implementation tools exist $[5,11,12]$. 
Attribute grammars have been used successfully in a few cases (for instance an Ada type checker [16] and a VHDL compiler [7]), but many people seem to have the impression that attribute grammars are clumsy, more complicated than the equivalent imperative programs. In particular, classical attribute grammars require dependencies between nodes that do not occur in the same production instance to be described through a chain of dependencies between neighboring nodes. The LIGA attribute grammar system [12] includes features that weaken this restriction, enabling, for example, a rule for a node to refer directly to an ancestral node by type. However, even this system does not schedule attribute grammars with dependencies between unrelated nodes in the tree.

Starting with Johnson and Fischer's proposal for "non-local" attribute grammars [9], several researchers have investigated the possibility of permitting direct non-local dependencies in attribute grammars. Such dependencies are induced by passing objects with fields through the attribute system. Elsewhere, the fields may be read, and (depending on the extension) other fields may be written. Since the places in the tree where the fields are written may be far removed from the places where they are read, these abilities are also called remote attribution [4]. Direct non-local dependencies increase the expressive power of attribute grammars, reduce the number of "copy rules" and, as reported by Hedin [8], permit more efficient (incremental) implementation.

Remote (direct non-local) dependencies require extensions to the standard attribute grammar analyses. If objects are added with only trivial extension to the analysis, then, depending on how the extension is formulated, standard analysis will either report spurious circularities, or (worse) fail to notice dependencies at all. In the latter case, we say that the analysis is unsafe. For example, in Augusteijn's Elegant system [2], a field in an object can be designated "lazy," thus permitting the object to be passed through the attribute system before the field has been assigned. No analysis ensures the field is assigned before it is used. With LIGA [12], objects can be created in multiple passes, but the description writer must direct the scheduling through the addition of dependencies involving extra control attributes. The implementation tool is unable to determine if the extra dependencies are sufficient. Hedin's door attribute grammars leave all responsibility for the scheduling of remote dependencies to hand-written code.

Johnson and Fischer described a simple technique that can handle some remote dependencies. It generates similar control attributes to those generated in this work, that is, in both directions paralleling the attribute carrying an object. But the technique relies upon trusted user-supplied directives, and also does not appear to work for remote writing of fields. In contrast, this paper develops an algorithm for analyzing remote dependencies that works automatically, and even where guided by hand-written directives, never yields an unsafe analysis.

The first section following this introduction gives a flavor of the expressive power provided by remote attribution. The next section describes our extended attribute grammars precisely. Section 4 describes algorithms that enable static scheduling of extended attribute grammars. The paper concludes with some suggestions for further research. 


\section{Remote Attribution}

In a classical attribute grammar, any values that need to be transmitted between remote nodes must be transmitted through the least common ancestor in the tree. Sometimes, several values may be "ready" at the same time and may be packaged up (in some sort of record) and transmitted together, but other times, packaging would cause a circular dependency and thus the values must be transmitted independently. Independent transmission increases the number of rules for an attribute grammar and, in the case of name resolution, may require multiple lookups with the same key in isomorphic dictionaries. The decision of whether two values can be packaged together (thus reducing complexity and increasing efficiency) relies on global scheduling information. This fact forces the attribute grammar writer to schedule by hand; an implementation tool can only verify that the schedule is correct (non-circular).

In this section, we describe an extension in which objects with fields may be created. References to these objects may be transmitted through the attribute system. When a reference to an object reaches any location in the tree, its fields may be read and written. Copy rules may be necessary to transmit references, but no additional copy rules are necessary. The fields of an object are scheduled automatically and safely, thus relieving the attribute grammar writer of doing the scheduling by hand. Thus remote attribution improves the ability of an attribute grammar to fulfill the task of declarative specification.

This section shows how a simple task may be accomplished in an attribute grammar, first with restricted classical attribute rules and then with the features provided by remote attribution. The task in question is static checking (name resolution and type-checking) for a simple programming language. Name resolution here refers to determining which declaration is being used for each identifier in an expression. The attribute grammars also determine when a declaration goes unused. In this example, the result of the attribution is a set of error messages.

Both attribute grammars include the complete abstract context-free grammar (shown in italics) for the programming language. Each program in the language consists of a block; each block has a sequence of variable declarations and then a sequence of statements. Variables are either of type integer or of type string. Statements are either nested blocks or assignment statements, where the expression being assigned must be of the same type as the expression being assigned to it. (Assume that the parser disallows assignments such as $1=2$.) Expressions can be constants or identifier uses.

\subsection{Static Checking with a Classical Attribute Grammar}

Figure 1 gives a classical attribute grammar for performing static checking. The first part of the attribute grammar defines the attributes for each nonterminal of the language. Each attribute is declared either inherited (inh) or synthesized (syn). Intuitively, the former attributes are "top-down" and the latter "bottom-up." A set of (oriented) attribute equations is specified for each 


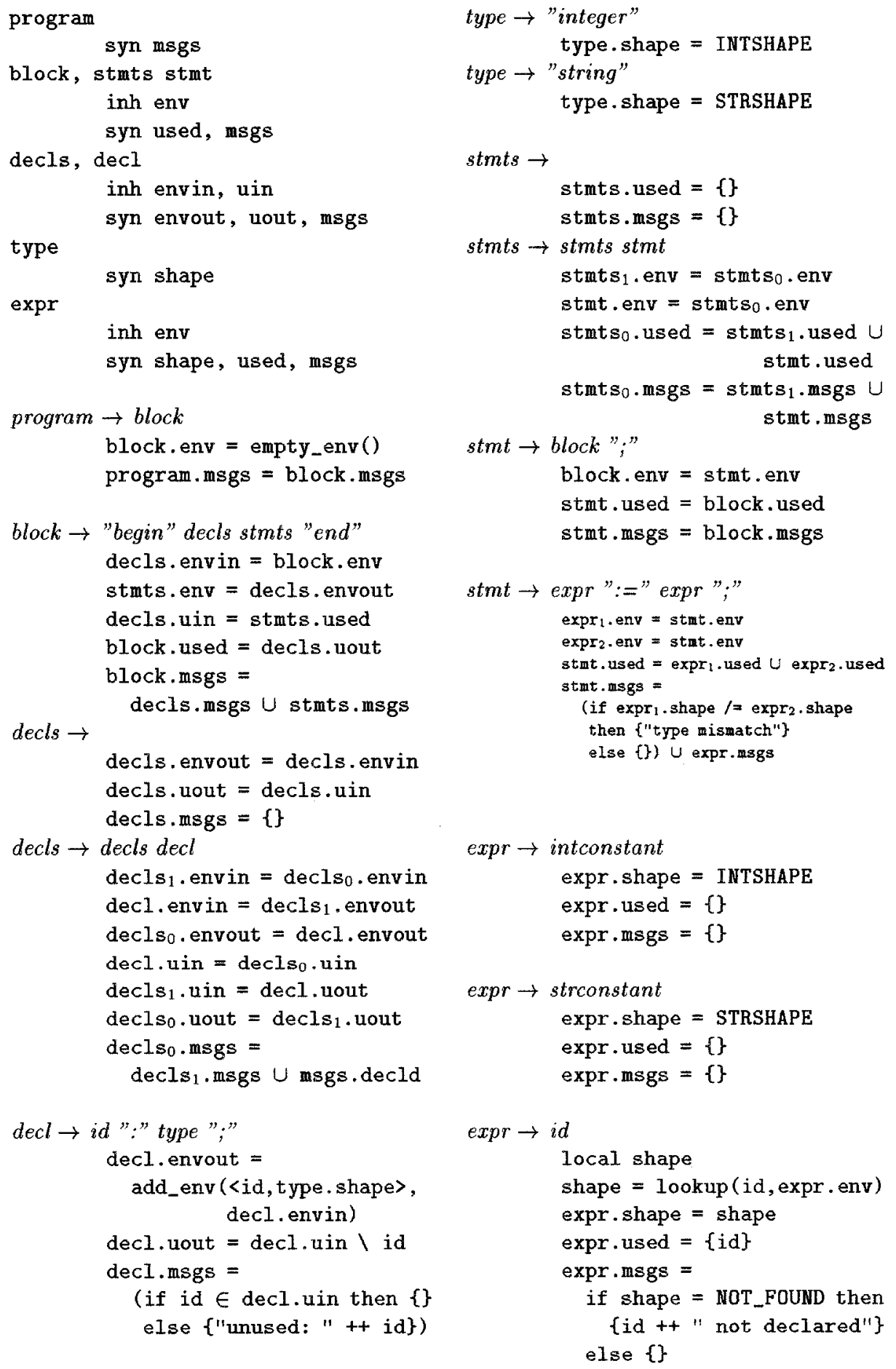

Fig. 1. A classical attribute grammar for static checking 
production. These equations define the synthesized attributes of the result of the production and the inherited attributes of the children of the production. Furthermore, for each production, an attribute grammar may define locals (also known as "local attributes"). A local is assigned in the same way as an attribute.

Much of the attribute grammar in Figure 1 concerns environments, sets of identifiers or error messages. These rules make the attribute grammar more complex, although practical attribute grammar systems such as LIGA [12] and FNC2 [11] have ways of expressing these rules more succinctly. More seriously, passing around sets of used identifiers and looking up identifiers in them implies an inefficiency that would not arise in an imperative description that passed around objects and set a used flag in the object when it is used.

\subsection{Static Checking Using Remote Attribution}

In Figure 1, the type (shape) of declaration (decl $\rightarrow$ id ":" type ";") is only put into the environment so that the type of a variable can be determined at a use site. The type of a variable does not influence the way in which names are resolved. Conceptually, an object representing declaration itself is what should be found and then any information needed about the declaration could be determined directly from the object rather than through the environment.

Figure 2 expresses this intuition. Each declaration inserts an object into the set of declarations local to its block. This object has two fields: shape records the type of the declarations and used records whether the variable declared is used anywhere. At the use site, the appropriate declaration is found in the declarations for the scope. The type of the expression depends on a use of the shape attribute of an object created remotely.

So far, remote attribution may seem to be contributing little. Not only must the type be fetched using indirection, but an extra rule is needed to create the object to be fetched. However, the dependencies are different. The list of declarations no longer depends on each declaration's shape. A general incremental evaluation strategy that could track remote dependencies would not have to look up every identifier again just because the type of one declaration changed.

Having the object representing the declaration available at the use site makes it easier to transmit further information. In this case, determining whether a declaration is used is extremely easy to specify. The object created for each declaration has a collection field named used. A collection field has a starting value (here false) and a combination function (here or) used to form a final value from all the definitions. Since the definitions are unordered, a combination function must be commutative and associative.

Collection fields are defined using $\sqsupseteq$ to emphasize the fact that only a partial attribute definition is being given. A use of a collection field will not be scheduled until all the definitions have been combined into a final value. Only two places in the attribute grammar handle whether declarations are used: where the declaration is looked up and where it is checked whether it is used. The situation in the classical attribute grammar is far more complicated, involving many different attributes passed around almost every node in the tree. 

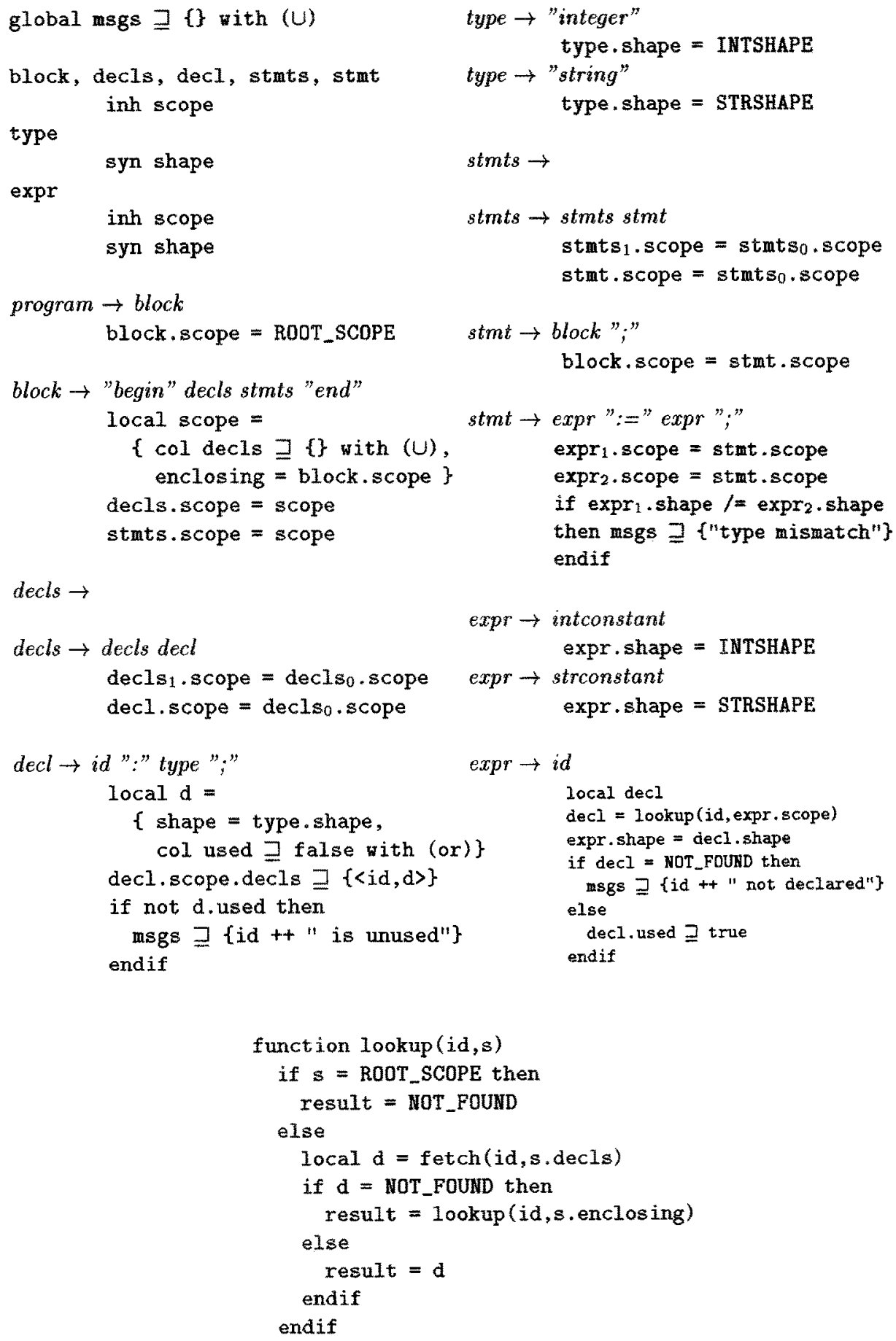

Fig. 2. Static checking using remote attribution 
A remotely defined field must be a collection field because there can be no guarantee that it will be defined precisely once. One could permit multiple definitions as long as they were identical, and have a default for when there were no definitions, but such exceptions are messy and are insufficient to handle similar cases. Suppose instead of knowing whether a declaration is used, one would like to know how many times it is used lexically. Directly incrementing the remote attribute would work in an imperative language but in a specification is ill-formed. Unfortunately, the classical attribute grammar formulation of what is expressed here is yet more complex than that for determining only whether a declaration is used. Using a collection field, the specification is both clear and declarative:

$$
\begin{aligned}
\mathrm{d}=\left\{\begin{array}{l}
\text { shape }=\text { type.shape } \\
\text { use_count } \supseteq 0 \text { with }(+)\}
\end{array}\right.
\end{aligned}
$$

decl.use_count $\sqsupseteq 1$

The use_count field has a starting value of 0 and definitions are added to create the final value.

In the classical attribute grammar, error messages are collected into sets passed to the root. The collection process clutters the attribute grammar and affects productions even when they do not generate any error messages of their own. Such tasks can be accomplished using global collections that are simply collection fields of a special global object created in the rules for the root production. A "sugared" notation makes these collection fields easier to use. Global collections generalize the error message feature of Olga [10].

Using remote attribution greatly reduces the amount of "busy-work" in attribute grammars. Previous extensions for reducing copy rules (such as the INCLUDING keyword in LIDO [12]) can further simplify both examples; for example, to directly copy the environment/scope from a block node to identifier occurrences in expressions. But by leaving the scheduling of object construction to the implementation tool, the attribute grammar in Figure 2 accomplishes the same task as that in Figure 1 at a higher level, and it does so without user-visible side-effects.

The examples in Figures 1 and 2 are not completely comparable. The first builds up an environment as a (sequential) list, the second stores it as an unordered set with an explicit "enclosing scope pointer." Remote definition cannot be used to create a list because the definitions are unordered. However, the relative position in some arbitrary linearization of the tree such as the sequence of nodes visited in a preorder traversal may be used to check whether a declaration is defined before it is used, or whether an identifier is multiply declared.

\section{Extended Attribute Grammars}

The extended attribute grammars described here borrow ideas from composable attribute grammars [6] (object declarations), conditional attribute gram- 
mars [3] (if clauses) and dynamic attribute grammars [15] (local instances of nonterminals). This combination of features was first proposed by Boyland [4] along with other extensions. Rather than permit direct manipulation of the nodes of the parse tree as in Boyland's APS, we follow the example of other work $[6,8,14]$ in requiring values to be packed in objects, references to which are passed through the attribute system. Attribute grammars using the first technique can be mechanically transformed into ones using the second with only minor change, and indeed objects provide a more powerful facility since the rules for a single production can include multiple object declarations.

User-defined functions (which may manipulate objects) are supported using local instances of nonterminals that are analyzed in the same manner as nonterminals in a (conditional) attribute grammar. This facility is fully general and permits us even to model functions with multiple return values. By performing this canonicalization, functions may be scheduled using the same machinery as that for (conditional) attribute grammars [15].

For simplicity, this paper assumes an untyped model: objects, attributes, locals, primitive operators are untyped. Also for simplicity, all collection fields are considered (primitive) bags: unordered collections of values.

\subsection{Definitions}

Assume we have several disjoint sets: $a \in A(X)$, the set of attributes of nonterminal $X$ (partitioned into $s \in S(X)$ and $i \in I(X)$, synthesized and inherited attributes), $f \in F$, the set of fields (partitioned into $d \in D$ and $c \in C$, direct assigned fields and remotely assigned collections), and $l \in L$ a set of locals (partitioned into $v \in V$ and $o \in O$, value locals and object locals). Let $A$ be the (disjoint) union of attributes for all nonterminals, and similarly for $S$ and $I$. Let $E$ be the set of expressions in one of the following forms:

$$
e \in E=X_{i} . a|v| g\left(v_{1}, \ldots, v_{n}\right)|\operatorname{id}(v)| \operatorname{ref}(o) \mid v . f
$$

(where in the first case, $i=0, a \in I$ or $i>0, a \in S$ ). Each $g$ is a primitive operator that does not examine contents of objects. Examples of primitive operators include addition of primitive integers, or the creation of a primitive pair. The transformations described in this section will make use of several primitive operators, for instance use, which takes multiple arguments and returns a value that combines its arguments in some way. The operator id returns a primitive value representing the identity of an object. All instances of objects have a unique identity. ${ }^{1} \mathrm{~A}$ reference to an object is computed as an attribute value using ref. A field $f$ is fetched from an object reference stored in $v$ with the syntax $v . f$.

The rules associated with each production have the following forms:

\footnotetext{
${ }^{1}$ Instead of using object identity, one could add a field id to every object that is initialized in a global traversal of the tree to unique integer values. Then id $(v)$ would be written $v$.id. We choose to use object identity since it permits a simpler efficient implementation.
} 


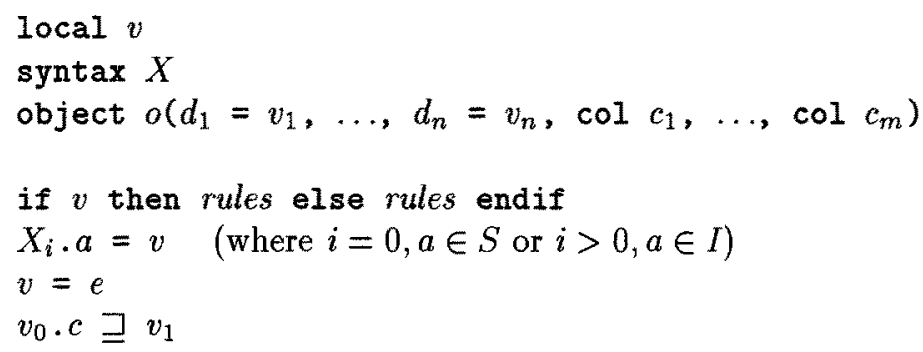

The first three forms declare names that are visible within the scope of the rule list they occur in. The first declares a new local value, the second a new instance of a nonterminal (treated as a child) and the third a new object. The object declaration also initializes the normal (non-collection) fields within the object. Define $D_{o}=\left\{d_{1}, \ldots, d_{n}\right\}$ and $C_{o}=\left\{c_{1}, \ldots, c_{m}\right\}$ the directly assigned and the collection fields of object $o$, and define $F_{o}=D_{o} \cup C_{o}$ its complete set of fields. For an object $o \in O$ and $d \in D_{o}$, define $v_{o d}$ to be the local value $v$ assigned to field $d$.

The next four forms of rules are executable rules. The first form is a conditional clause [3]. The next two forms of executable rules describe definitions of attributes of nonterminals in productions and locals. The last form is a definition of the collection field $c$ of the object whose reference is carried by $v_{0}$. The syntax here requires many local values; in examples, we use a less restrictive syntax:

- a local may be assigned in its declaration;

- an expression may be used in the place of the use of a local value;

- braces \{\} may be used in an expression:

$$
v_{0}=\left\{d_{1}=v_{1}, \ldots, d_{n}=v_{n}, \operatorname{col} c_{1}, \ldots, \operatorname{col} c_{m}\right\}
$$

has the same meaning as

$$
\begin{aligned}
& \text { object } o\left(d_{1}=v_{1}, \ldots, d_{n}=v_{n}, \operatorname{col} c_{1}, \ldots, \operatorname{col} c_{m}\right) \\
& v_{0}=\operatorname{ref}(o)
\end{aligned}
$$

for some fresh object $o$;

- syntax declarations may include definitions of inherited attributes:

$$
\text { syntax } s\left(a_{1}=v_{1}, \ldots, a_{n}=v_{n}\right)
$$

has the same meaning as

$$
\begin{aligned}
& \text { syntax } s \\
& \text { s. } a_{1}=v_{1} \\
& \vdots \\
& \text { s. } a_{n}=v_{n} .
\end{aligned}
$$

\section{Analysis and Transformation of Remote Attribution}

In this section, we develop an analysis technique for extended attribute grammars that enables a translation to conditional attribute grammars. Then the standard techniques for analyzing and implementing conditional attribute grammars can 
be applied. Extended attribute grammars can thus have membership in standard evaluation classes such as strongly noncircular (SNC) or ordered (OAG) attribute grammars using the algorithms for (conditional) attribute grammars described in earlier work [3].

For explanatory purposes, we first start with a simple form of the analysis that does not handle collections. Then we show how extended attribute grammars with collections can be transformed into ones without collections. This transformation is then combined with the simple analysis to give a more powerful analysis. Next we discuss finite approximations of the analysis domain that ensure termination. The result is a powerful and sophisticated analysis that can be used as part of a tool that implements our running example.

\subsection{Analysis of remote field selection}

The key intuition behind the analysis is that a reference to an object can be created before all of its fields are defined. The work that the analysis technique performs ensures that field selection operations are not performed until the corresponding field is defined.

At any place where a remote field selection occurs in the extended attribute grammar being analyzed, the transformed rule will contain a dependence on a control attribute. This attribute represents the information that the field has been defined. It parallels the attribute carrying the reference to the object but carries no runtime value in the implementation and is used only for scheduling purposes. There are potentially multiple generated control attributes. As well as one for every field, each field may itself carry references to objects, and so control attributes will be needed for the fields of these fields. In general, there may be a generated control attribute for every nonempty field selection path (sequence of field names). All of the generated control attributes are called fibers because one may think of the entire, fully defined object as a rope consisting of all these control attributes bundled together.

Of course, there may be an infinite number of such paths and thus the analysis "algorithm" described in this section may not terminate. In order to prepare for approximation techniques described in Section 4.3, our analysis will be defined over any set $\Phi^{*}$ of abstract field selection paths (or abstract paths) that abstracts the infinite set $F^{*}$ of all concrete field selection paths. In general, each abstract path may represent multiple (even infinite) concrete paths. The abstract set must include the empty field selection path $\epsilon \in F^{*}$; the empty path represents the object itself. The abstraction is specified through the definition of an abstract operation, path lengthening (denoted $f \cdot \phi$ ), that takes a field $f \in F$ and an abstract path $\phi \in \Phi^{*}$ and returns a set of abstract paths. Path shortening (denoted $f^{-1} \cdot \phi$ ) is defined in terms of lengthening:

$$
f^{-1} \cdot \phi=\left\{\phi^{\prime} \mid \phi \in f \cdot \phi^{\prime}\right\} \text {. }
$$

Both operations are extended to operate on sets of abstract paths: $f \cdot \Phi=$ $\bigcup_{\phi \in \Phi} f \cdot \phi$, and $f^{-1} \cdot \Phi=\bigcup_{\phi \in \Phi} f^{-1} \cdot \phi$. 


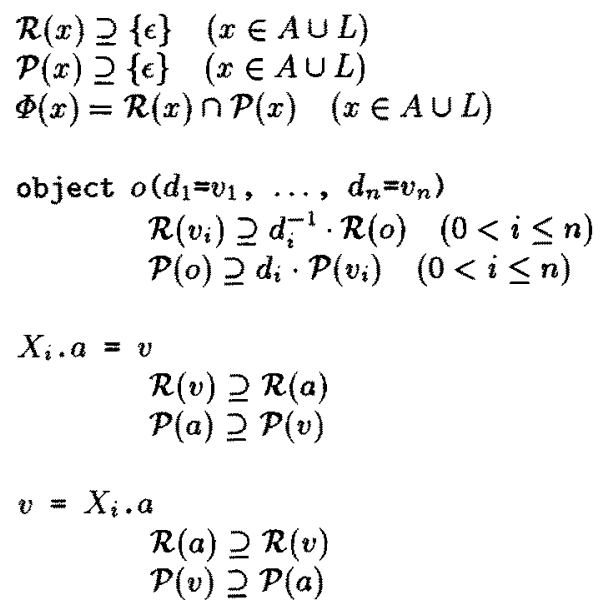

$$
\begin{aligned}
& v_{0}=v_{1} \\
& \mathcal{R}\left(v_{1}\right) \supseteq \mathcal{R}\left(v_{0}\right) \\
& \mathcal{P}\left(v_{0}\right) \supseteq \mathcal{P}\left(v_{1}\right) \\
& v_{0}=g\left(v_{1}, \ldots, v_{n}\right) \\
& \mathcal{R}\left(v_{i}\right) \supseteq \mathcal{R}\left(v_{0}\right) \quad(0<i \leq n) \\
& \mathcal{P}\left(v_{0}\right) \supseteq \mathcal{P}\left(v_{i}\right) \quad(0<i \leq n) \\
& v=\operatorname{ref}(o) \\
& \mathcal{R}(o) \supseteq \mathcal{R}(v) \\
& \mathcal{P}(v) \supseteq \mathcal{P}(o) \\
& v_{0}=v_{1} \cdot f \\
& \mathcal{R}\left(v_{1}\right) \supseteq f \cdot \mathcal{R}\left(v_{0}\right) \\
& \mathcal{P}\left(v_{0}\right) \supseteq f^{-1} \cdot \mathcal{P}\left(v_{1}\right)
\end{aligned}
$$

Fig. 3. Analysis of remote field selection.

The task of analysis is to determine which fibers must be generated, and then the task of the transformation is to generate a (conditional) attribute grammar without remote field selection that uses fiber dependencies to ensure a valid schedule. The analysis computes a set of field selection paths $\Phi(x)$ for every attribute or local $x \in A \cup L$. A backward, demand-driven analysis determines what field selections are performed on objects whose references are carried by $x$. This set is called $\mathcal{R}(x) \subseteq \Phi^{*}$, the required field selection paths. A forward analysis determines what field selection paths are possible for objects carried by $x$. This set is called $\mathcal{P}(x) \subseteq \Phi^{*}$, the provided field selection paths. The set used for scheduling is the intersection of these two sets. (Intersection makes the analysis more precise, but is otherwise unnecessary. When we consider collections, the additional precision plays a crucial role.)

Figure 3 shows a set constraint system, for which the least fixed point solution is to be found. The first two constraints initialize each set to contain the empty path. In the first constraint for object declarations, the required paths for each field's local is made to contain any required path of the whole object that starts with that particular field after stripping off the field. The next constraint collects the paths provided by each field's local after appending the fields. The next five pairs of constraints show that required and provided fields are propagated backward and forward, respectively. The first constraint for field selection shows how a path $f$ is added to a required set: $\epsilon \in \mathcal{R}\left(v_{0}\right)$ is known, and thus $\mathcal{R}\left(v_{1}\right) \supseteq$ $f \cdot \epsilon$. The last constraint shows how paths provided by $v_{1}$ can be provided by $v_{0}$ after the prefix $f$ is removed. There are no constraints for $v_{0}=\operatorname{id}\left(v_{1}\right)$.

If these constraints have a finite least fixed point solution, we can perform a transformation (see Figure 4) that adds control attributes $a \$ \phi(a \in A)$ for each $\phi \in \Phi(a)$, and new locals $l \$ \phi(l \in L)$ for each $\phi \in \Phi(l)$ and associated equations. 


$$
\begin{aligned}
& \phi \epsilon^{\prime} \Phi \equiv \phi \in \Phi \wedge \phi \neq \epsilon \\
& \text { object } o\left(d_{1}=v_{1}, \ldots, d_{n}=v_{n}\right) \\
& \text { o\$ } \$=\operatorname{use}\left(v_{i} \$ \phi^{\prime} \mid \phi^{\prime} \in d_{i}^{-1} \cdot \phi, 0<i \leq n\right) \quad(\phi \in \Phi(o)) \\
& X_{i} \cdot a=v \\
& X_{i}, a \$ \phi=v \$ \phi \quad\left(\phi \epsilon^{\prime} \Phi(a) \cap \Phi(v)\right) \\
& v=X_{i} \cdot a \\
& v \$ \phi=X_{i}, a \$ \phi \quad\left(\phi \in^{\prime} \Phi(v) \cap \Phi(a)\right) \\
& v_{0}=v_{1} \\
& v_{0} \$ \phi=v_{1} \$ \phi \quad\left(\phi \in^{\prime} \Phi\left(v_{0}\right) \cap \Phi\left(v_{1}\right)\right) \\
& v_{0}=g\left(v_{1}, \ldots, v_{n}\right) \\
& v_{0} \$ \phi=\operatorname{use}\left(v_{i} \$ \phi \mid \phi \in^{\prime} \Phi\left(v_{i}\right)\right) \quad\left(\phi \in^{\prime} \Phi(v)\right) \\
& v=\operatorname{ref}(o) \rightarrow v=\operatorname{prog} 1(\operatorname{ref}(o), o \$ \epsilon) \\
& v \$ \phi=o \$ \phi \quad\left(\phi \in^{\prime} \Phi(v) \cap \Phi(o)\right) \\
& v_{0}=v_{1} \cdot f \longrightarrow v_{0}=\operatorname{select}_{f}\left(\dot{i d}\left(v_{1}\right), \text { use }\left(v_{1} \$ \phi \mid \phi \in f \cdot \epsilon\right)\right) \\
& v_{0} \$ \phi=\operatorname{use}\left(v_{1} \$ \phi^{\prime} \mid \phi^{\prime} \in f \cdot \phi\right) \quad\left(\phi \epsilon^{\prime} \Phi\left(v_{0}\right)\right)
\end{aligned}
$$

Fig. 4. Transformation of remote field selection

As a special case, $x \notin \epsilon$ is the same as $x$, except for $x \in O$. The last two forms of rules, creating object references, and field selection have the most interesting translations. Since object references are assumed not to depend on any of the fields, we need to add direct dependencies in the case that the abstraction ignores the field. The local control attribute $o \$ \epsilon$ carries the necessary dependencies (prog 1 returns its first value). Field selection is made to depend on all fibers (typically there is only one) for the abstract paths $f \cdot \epsilon$. The primitive operation select $f$ performs the actual field selection.

\subsection{Analyzing collection assignments}

In this section, we first describe a reduction of extended attribute grammars with collections to those without. This reduction introduces serious circularities that render it useless for direct implementation. But we show how the reduction can be combined with the analysis and transformation described in the previous section into a compound analysis that handles both remote field selection and remote collection assignment.

The reduction is described as the sequence of two stages: first collection fields are transformed into normal fields with the introduction of local collections and attribute collections; next local collections and attribute collections are transformed into normal locals and attributes. The intermediate attribute grammar will have locals and attributes that can be assigned multiply with all the results going into a bag, as with collections fields. However, collection locals and 


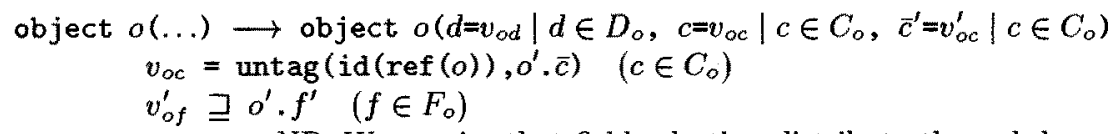

NB: We require that field selection distribute through bags.

$$
\begin{array}{cc}
X_{i} \cdot a=v & v_{0}=g\left(v_{1}, \ldots, v_{n}\right) \\
v^{\prime} \sqsupseteq X_{i} \cdot a^{\prime} & v_{i}^{\prime} \sqsupseteq v_{0}^{\prime} \quad(1 \leq i \leq n) \\
v=X_{i} \cdot a & v_{0}=\operatorname{ref}(o) \\
X_{i} \cdot a^{\prime} \sqsupseteq v^{\prime} & o^{\prime} \sqsupseteq v_{0}^{\prime} \\
v_{0}=v_{1} & v_{0}=v_{1} \cdot f \\
v_{1}^{\prime} \sqsupseteq v_{0}^{\prime} & v_{1}^{\prime} \sqsupseteq \operatorname{bag}\left(\left\{f^{\prime}=v_{0}^{\prime}\right\}\right) \\
v_{0} \cdot c \sqsupseteq v_{1} \longrightarrow v_{0}^{\prime} \sqsupseteq \operatorname{bag}\left(\left\{\bar{c}=\operatorname{tag}_{c}\left(\operatorname{id}\left(v_{0}\right), v_{1}\right)\right\}\right) &
\end{array}
$$

Fig.5. Transformation from collection fields to collection locals and attributes.

attributes are not assigned remotely, and so it is a simple matter to convert their assignments into regular assignments. Collection locals and attributes are assigned using $\sqsupseteq$, so we add the following new permitted forms for our intermediate attribute grammars:

$$
\begin{aligned}
& v_{0} \sqsupseteq v_{1} \\
& X_{i} \cdot a \sqsupseteq v
\end{aligned}
$$

The intermediate attribute grammar includes all the elements of the original, with an additional attribute $a^{\prime}$ for each $a \in A$ and an additional local value $l^{\prime}$ for each $l \in L$. These new attributes and locals track the data dependencies in the opposite direction; they carry values from the remote collection assignments back to the object being assigned. Additionally for every $o \in O$ and $c \in C$, we introduce two new locals $v_{o c}$ and $v_{o c}^{\prime}$ that will collect the values coming back from the various remote collection assignments. We also introduce new fields $d^{\prime}$ for every field $d \in D$, and new fields $c^{\prime}, \bar{c}, \bar{c}^{\prime}$ for every $c \in C$. In the intermediate attribute grammar, all these fields are normal (not collection) fields. Figure 5 shows what new rules are generated for each rule in the original.

The new (or reverse) locals, attributes and fields carry information about collection field assignments to objects transmitted by the original local, attribute or field. For example, consider the transformation of $v_{0} . c \sqsupseteq v_{1}$ in Figure 5 . The transformed rule ensures that the reverse local $v_{0}^{\prime}$ carries a bag with (at least) one object with a field $\bar{c}$ bound to a tagging of the value of $v_{1}$. Ignoring the tagging, one sees that the reverse local $v_{0}^{\prime}$ is made to depend on the value $v_{1}$ assigned to the collection field. Assuming the reference to the object whose collection field is assigned was transmitted by simple copy rules from a rule $v_{0}=$ ref $(0)$, the reverse attributes and locals transmit the bag containing the object with the 
tagged assignment back to $o^{\prime}$ where the tagged assignments are selected (using $o^{\prime} . \bar{c}$ ) and then the tag is examined (using untag) and only the assignments for the object in question are stored in $v_{o c}$, which is used to initialize the $c$ field of the object. Again, ignoring tagging, one sees that the $c$ field is made to depend on the value assigned to it remotely.

The reverse fields $f^{\prime}$ are used because some objects whose collection fields are assigned are fetched from other objects. The rule for field selection $v_{0}=v_{1} \cdot f$ in Figure 5 shows how reverse fields are used. The reverse local $v_{1}^{\prime}$ is made to carry an object that carries assignments to objects fetched from the $f$ field; this rule uses the reverse field $f^{\prime}$ to distinguish these assignments from assignments to the field $f$ itself. Then, assuming the object with the $f$ field was computed only using copy rules, we can trace the object back to a rule $v_{0}=\operatorname{ref}(o)$. The reverse local $o^{\prime}$ will contain an object with field $f^{\prime}$ containing the assignments of collection fields of objects fetched through the $f$ field of $v_{0}$. These assignments need to be transmitted back to the objects referenced through the $f$ field. If $f$ is a normal field of the object $\left(f=d \in D_{o}\right.$ ), this task is accomplished by passing the assignments in $o^{\prime} \cdot d^{\prime}$ to the reverse local $v_{o d}^{\prime}$ (the reverse of $v_{o d}$ ). If $f$ is a field not stored in the object $\left(f \notin F_{o}\right)$, the set is clearly irrelevant and can be ignored.

If $f$ is a collection field ( $f=c \in C_{0}$ ), then the (tagged) assignments being carried by $o^{\prime} \cdot c^{\prime}$ are for objects that were themselves remotely assigned into $c$. Thus they need to be transmitted back to all the points where $c$ is remotely assigned. It might seem that reversed reverse locals and attributes are needed, but instead the task is neatly accomplished by transmitting the assignments in a field $\left(\bar{c}^{\prime}\right)$ of the original object, through the mediation of $v_{o c}^{\prime}$. Then at every collection assignment $v_{0} \cdot c \sqsupseteq v_{1}$ the reverse local $v_{1}^{\prime}$ is assigned to value transmitted in this field, as seen in Figure 5.

Once we have performed the translation described in Figure 5 , we have effectively given a semantics to collection fields using collection locals and attributes. The intermediate attribute grammar is then transformed into an extended attribute grammar without collections by assigning a collection attribute or local to the bag union of all the values it is assigned.

Unfortunately, the reduction introduces serious circularities. Every attribute transmitting an object directly or indirectly must be evaluated before any collection field of this object can be computed. The solution to this difficulty lies in the observation that a correct schedule for remote selection only requires the translation to depend on the control attribute for the field selected, and not on the object itself, which is needed only for implementation. For the case of remote field assignment, the (introduced) object carrying the assignment in the reverse direction is not needed for implementation. The actual values of reverse attributes and locals are not needed. Therefore in the combined analysis that handles both remote field selection and collection assignment, we do not initialize the require and provide sets for reverse attributes and locals to include $\epsilon$. Similarly, the combined translation differs from the composition of the reduction with the transformation for remote field selection by treating generated field selections specially. 


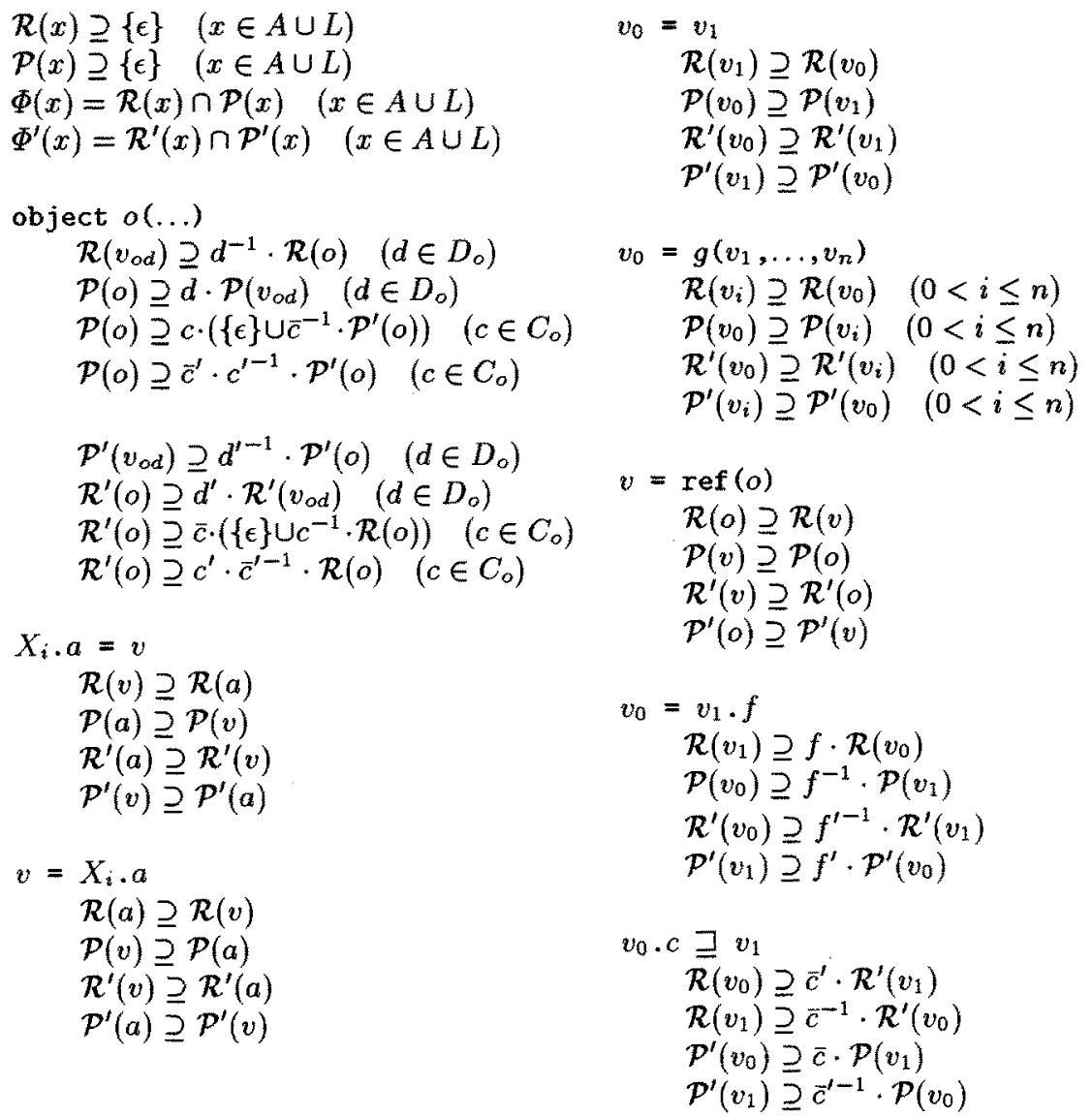

Fig. 6. Analysis of extended attribute grammars.

Figure 6 gives the combined analysis; a new set $\mathcal{R}^{\prime}(x)$ which evaluates to what $\mathcal{R}\left(x^{\prime}\right)$ would be for the intermediate form, and similarly with $\mathcal{P}^{\prime}(x)$ and $\mathcal{P}\left(x^{\prime}\right)$. The combined translation is given in Figure 7 .

\subsection{Approximation}

If we use the analysis described with concrete (unabstracted) field selection paths, it does not always terminate and in fact does not terminate on our running example, the extended attribute grammar in Figure 2. We end up with longer and longer paths with arbitrarily long chains of enclosing field selections, for example: enclosing enclosing ... enclosing decls shape. In this section, we describe finite abstractions $\Phi^{*}$ of the infinite set $F^{*}$ that permit a terminating analysis. One elegant trivial case is $\Phi^{*}=\{\epsilon\}$ where lengthening always returns 


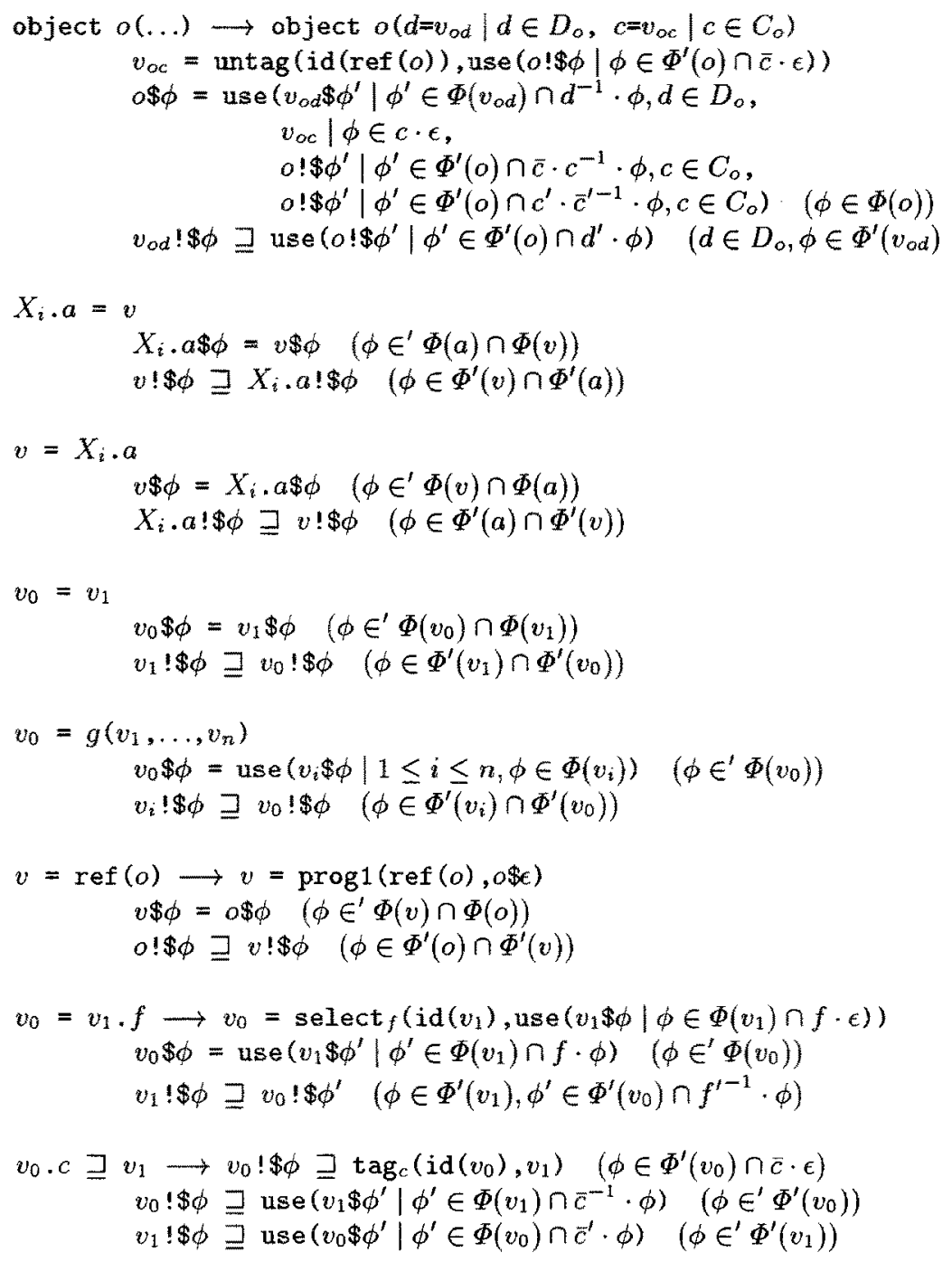

Fig. 7. Transformation of extended attribute grammars.

$\{\epsilon\}$, yielding the conservative analysis that a reference to an object depends on all of its fields. Collection fields induce circularities.

There are two approaches an analysis tool may adopt for approximation: define an approximation for all attribute grammars; or, let the user declare an approximation (from a class of approximations). The first approach suffers from the difficulty of defining an approximation that is powerful enough to handle most reasonable examples without being so complex that analysis takes too long. The second approach can put too much burden on the user.

We have been experimenting with the second approach, keeping the specifi- 
cation simple by restricting the class of approximations. We ensure termination by picking as our finite set $\Phi^{*}$ the paths of $\left(F \cup F^{\prime}\right)^{*}$ with no duplicate fields. The approximation ignores the distinction between $c$ and $\bar{c}$ and between $c^{\prime}$ and $\bar{c}^{\prime}$. The description writer can specify two subsets of $F: F_{0}$, fields ignored during the analysis (for $f \in F_{0}, f \cdot \phi=f^{\prime} \cdot \phi=\{\phi\}$ ); $F_{1}$, fields permitted to repeat in sequence (for $f \in F_{1}, f \cdot f \phi=\{f \phi\}, f^{\prime} \cdot f^{\prime} \phi=\left\{f^{\prime} \phi\right\}$ ). In all other cases of lengthening, we prevent duplication: if the abstract path $f \cdot \phi$ is requested and $f$ already appears in $\phi$, then analysis is terminated with an error message. Other than for fields in $F_{0}$ and $F_{1}$, shortening is the same as for concrete paths.

A description writer should only put a field into $F_{0}$ if the value it will have can be computed at the same time as the object in which it occurs. Collection fields should not occur in $F_{0}$, because we need to broadcast the identity of the object to all the assignments of the collection field before we can compute the collection value. A field should be placed in $F_{1}$ if the value it refers to is the same "type" as the object in which it occurs. The only reason why all the rest of the fields $F-F_{0}$ are not placed in $F_{1}$ is for the precision of shortening.

I have a prototype implementation that performs fiber analysis for a subset of APS [4]. It also determines whether the description can be scheduled as a conditional ordered attribute grammar. The example in Figure 2 can be analyzed and scheduled using the approximation $F_{0}=\{$ shape,INTSHAPE, STRSHAPE, ROOT_SCOPE, NOT_FOUND $\}$, and $F_{1}=$ \{enclosing $\}$. In particular, here are the sets for the scope attribute for the decl nonterminal:

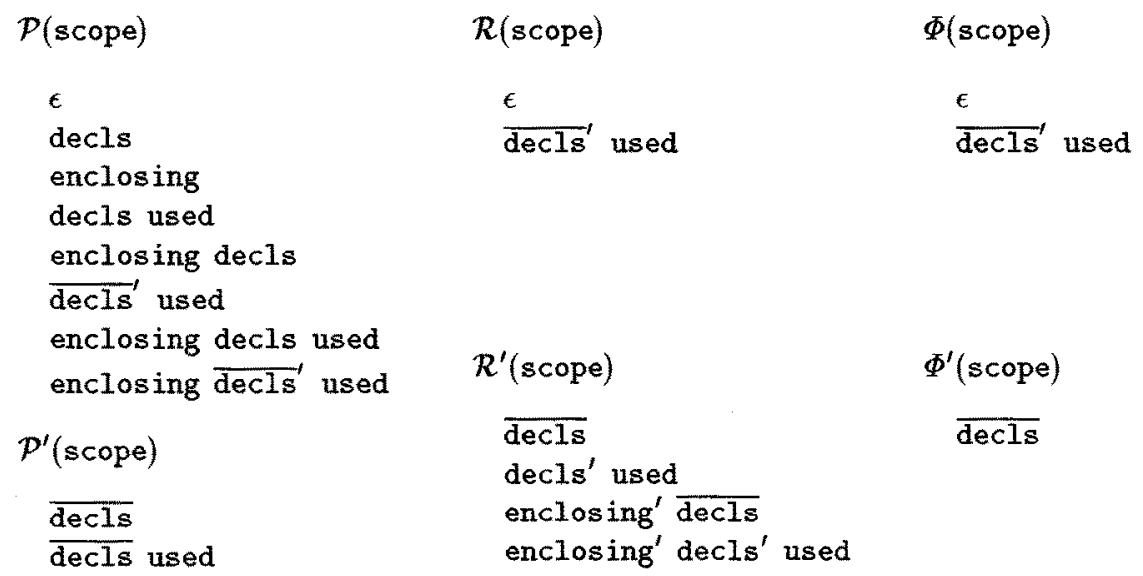

The resulting partition for $d e c l$ has two visits: $G$, scope $\rightarrow$ scope $\$ \$$ decls and scope\$decls'used $\rightarrow \mathrm{G} ! \$$ msgs. In the first visit, the implicit object representing shared global values such as INTSHAPE is required. The inherited attribute scope is also required. The result of this visit is an assignment of the decls collection of the scope. In the second visit, the control attribute representing all assignments of used collections is required. During the second visit a message may be added to the shared global collection msgs. This visit does not involve passing any true values and is executed purely for side-effect. The nonterminal decls also requires two visits. All other nonterminals need only a single visit.

A different approximation can give different results. If enclosing is put in 
$F_{0}$ instead, the result can still be scheduled, but in this case some nonterminals (such as block and $s t m t$ ) require three visits. Since the difference between a scope and the enclosing scope is ignored, the generated compiler cannot make use of the fact that a declaration in the simple language is only used within the block in which it is declared. In the first visit for a statement, decls in nested blocks are placed in their scope; in the second, names are looked up; and only in the third visit can we collect the error messages about unused declarations.

\section{Further Work and Conclusion}

Extended attribute grammars can be used to construct circular structures. The analysis algorithms can be used to safely schedule the construction, but traversal of the circular structure usually leads to cycles in the generated classical attribute grammar. These cycles are semantically uninteresting because they occur solely among control attributes and do not represent true scheduling conflicts. A technique developed by the author and Maddox [14, Chapter 10] can be applied in this case to remove the circularities.

So far, the analysis described in this paper has only been applied to small examples. More experience is needed in both application (to larger problems) and in implementation in order to determine the useful scope of the analysis.

In conclusion, extending attribute grammars with objects that can be transmitted independently of their fields makes it simpler to express certain compilerrelated tasks in a declarative framework. This paper describes the first generally applicable analysis technique for scheduling evaluation in attribute grammars extended with these capabilities.

\section{Acknowledgements}

This work grew out of a series of extended discussions with Bill Maddox. Rodney Farrow kindly sent us an unpublished manuscript of an analysis for remote field selection including a formalization of fiber approximation. Martin Jourdan and Didier Parigot at INRIA gave me the first opportunity to present this work. Many people have assisted me by reading and commenting on drafts.

\section{References}

1. H. Alblas. Attribute evaluation methods. In H. Alblas and B. Melichar, editors, Attribute Grammars, Applications and Systems. International Summer School SAGA Proceedings, Prague, Czechoslovakia, June 4-13, pages 48-113. SpringerVerlag, Berlin, 1991.

2. Lex Augusteijn. The Elegant compiler generator system. In Pierre Deransart and Martin Jourdan, editors, Attribute Grammars and their Applications. International Conference WAGA Proceedings, Paris, France, September 19-21, pages 238-254. Springer-Verlag, Berlin, 1990. 
3. John Tang Boyland. Conditional attribute grammars. ACM Transactions on Programming Languages and Systems, 18(1):73-108, January 1996.

4. John Tang Boyland. Descriptional Composition of Compiler Components. PhD thesis, University of California, Berkeley, 1996. Available as technical report UCB//CSD-96-916.

5. Rodney Farrow. The Linguist translator-writing system. Technical report, Declarative Systems Inc., June 1989.

6. Rodney Farrow, T. J. Marlowe, and D. M. Yellin. Composable attribute grammars: Support for modularity in translator design and implementation. In Conference Record of the Nineteenth Annual ACM SIGACT/SIGPLAN Symposium on Principles of Programming Languages, pages 223-234. January 1992.

7. Rodney Farrow and Alec G. Stanculescu. A VHDL compiler based on attribute grammar methodology. In Proceedings of the ACM SIGPLAN '89 Conference on Programming Language Design and Implementation, Portland, Oregon, USA, ACM SIGPLAN Notices, 24(7):120-130, July 1989.

8. Görel Hedin. An overview of door attribute grammars. In Peter A. Fritzson, editor, Proceedings of Compiler Construction, 5th International Conference, CC'9/, Edinburgh, U.K., pages 31-51. Springer-Verlag, Berlin, April 1994.

9. Gregory F. Johnson and Charles N. Fischer. A meta-language and system for nonlocal incremental attribute evaluation in language-based editors. In Conference Record of the Twelfth ACM Symposium on Principles of Programming Languages, New Orleans, Louisiana, USA, pages 141-151. ACM Press, New York, January 1985.

10. Martin Jourdan, Carole Le Bellec, and Didier Parigot. The OLGA attribute grammar description language: Design, implementation and evaluation. In Pierre Deransart and Martin Jourdan, editors, Attribute Grammars and their Applications. International Conference WAGA Proceedings, Paris, France, September 19-21, pages 222-237. Springer-Verlag, Berlin, 1990.

11. Martin Jourdan and Didier Parigot. Internals and externals of the FNC-2 attribute grammar system. In H. Alblas and B. Melichar, editors, Attribute Grammars, Applications and Systems. International Summer School SAGA Proceedings, Prague, Czechoslovakia, June 4-13, pages 485-504. Springer-Verlag, Berlin, 1991.

12. Uwe Kastens. Attribute grammars in a compiler construction environment. In H. Alblas and B. Melichar, editors, Attribute Grammars, Applications and Systems. International Summer School SAGA Proceedings, Prague, Czechoslovakia, June 4-13, pages 380-400. Springer-Verlag, Berlin, 1991.

13. Donald E. Knuth. Semantics of context free languages. Mathematical Systems Theory, 2(2):127-145, June 1968. Corrections appear in Mathematical Systems Theory, 5(1):95-96, 1971.

14. William Maddox. Incremental Static Semantic Analysis. PhD thesis, University of California, Berkeley, 1997.

15. Didier Parigot, Gilles Roussel, Martin Jourdan, and Etienne Duris. Dynamic attribute grammars. In Herbert Kuchen and S. Doaitse Swierstra, editors, International Symposium on Programming Languages, Implementations, Logics and Programs (PLILP '96), volume 1140 of Lecture Notes in Computer Science, pages 122-136. Springer-Verlag, Berlin, 1996.

16. J. Uhl, S. Drossopoulou, G. Persch, G. Goos, M. Dausmann, G. Winterstein, and W. Kirchgässner. An Attribute Grammar for the Semantic Analysis of Ada, volume 139 of Lecture Notes in Computer Science. Springer-Verlag, Berlin, 1982. 